\title{
Examining plasma microRNA markers for colorectal cancer at different stages
}

\author{
Yan Sun ${ }^{1,5}$, Yuexin Liu ${ }^{1}$, David Cogdell ${ }^{1}$, George A. Calin ${ }^{2,4}$, Baocun Sun ${ }^{5}$ Scott \\ Kopetz $^{3}$, Stanley R. Hamilton ${ }^{1}$, Wei Zhang ${ }^{1,4}$ \\ ${ }^{1}$ Department of Pathology, The University of Texas MD Anderson Cancer Center, Houston, TX 77030, USA \\ ${ }^{2}$ Department of Experimental Therapeutics, The University of Texas MD Anderson Cancer Center, Houston, TX 77030, USA \\ ${ }^{3}$ Department of Gastrointestinal Medical Oncology, The University of Texas MD Anderson Cancer Center, Houston, TX 77030, \\ USA \\ ${ }^{4}$ The Center for RNA Interference and Non-Coding RNAs, The University of Texas MD Anderson Cancer Center, Houston, \\ TX 77030, USA \\ ${ }^{5}$ Department of Pathology, Tianjin Medical University Cancer Institute and Hospital, Tianjin 300060, China \\ Correspondence to: Wei Zhang, e-mail: wzhang@mdanderson.org \\ Yan Sun, e-mail: sunyan@tjmuch.com
}

Keywords: microRNA, biomarker, plasma, colorectal cancer, stage

Received: September 23, 2015

Accepted: January 23, 2016

Published: February 04, 2016

\section{ABSTRACT}

Circulating microRNAs (miRNAs) have emerged as promising biomarkers; however, few miRNAs have been reproducible and can be used in clinical practice. In this study, we screened the levels of $\mathbf{7 5 4}$ miRNAs using TaqMan array in $\mathbf{5 0}$ individual plasma samples from $\mathbf{1 0}$ demographically matched healthy controls and $\mathbf{4 0}$ colorectal cancer (CRC) patients (10 each of stage I-IV) and identified 22 miRNAs associated with the presence of and stages of CRC. Then we performed the validation for 11 miRNAs in an independent cohort including 187 CRC cases and 47 healthy controls. Comprehensive analyses showed that plasma miR-96 distinguished stage I-IV CRC from healthy controls with an area under curve (AUC) of $0.740 ;$ miR-203 separated stage III-IV CRC patients from stage I-II with an AUC of 0.757; and miR-141 differentiated stage IV CRC from stage I-III patients with an AUC of 0.851 . Survival analyses showed that plasma miR-96 and miR-200b were independent prognostic factors for overall survival. Thus, we propose four miRNAs (miR-96, miR-203, miR-141 and miR-200b) as clinically validated circulating biomarkers for CRC prognosis that warrant further evaluation for clinical utility.

\section{INTRODUCTION}

Colorectal cancer (CRC) remains the third most commonly diagnosed cancer in both men and women and it is the third leading cause of cancer death in the United States: an estimated 136,830 people were diagnosed and 50,310 people died of the disease in 2014 [1]. The main challenges in reducing the mortality rate are that $\mathrm{CRC}$ is asymptomatic in the early stages, there is no effective method of monitoring recurrence after treatment for early-stage CRC, and treatment for recurrent and metastatic CRC is suboptimal. Colonoscopy screening has contributed to the early detection of CRC and a decrease in mortality in recent years [2]. However, the invasive nature and relatively high cost of the procedure have hampered its application globally. Fecal occult blood test, although less invasive, has lower sensitivity (23.9\%) [3]. Screening with carcinoembryonic antigen (CEA) levels in the blood also has poor sensitivity (36-74\%, based on CRC stage) [4]. Therefore, the identification and validation of non-invasive circulating markers for CRC detection, monitoring, and prognosis remain an incomplete aspect of CRC research.

MicroRNAs (miRNAs) are small, non-coding RNAs that regulate the expression of target genes through an RNA-interfering mechanism or translational inhibition [5]. It is well established that aberrant expression of miRNAs is associated with cancer development, progression and treatment $[6,7]$. A number of studies have identified some miRNAs as potential circulating biomarkers for the diagnosis and prediction of CRC (Table S1) [8-23]. However, few miRNAs have been reproducible among 
studies. This discordance may be because most studies had a limited sample size, did not include CRC patients with all major clinical stages of disease, evaluated only a limited number of candidate miRNAs, and used different assay methodologies and normalizers.

To overcome the limitations of existing studies of circulating miRNAs in patients with CRC, we performed a two-step discovery and clinical validation study with a comprehensive statistical analysis in a large number of patients with all stages of CRC and in healthy controls. The design of this study is shown in the flow diagram in Figure 1. In the first step, we measured the levels of 754 miRNAs in 50 plasma samples, including 10 healthy controls and 10 stage I, 10 stage II, 10 stage III, and 10 stage IV CRC patients. On the basis of an analysis to select suitable internal reference miRNAs, we evaluated plasma miRNAs that had potential for the detection of the presence of CRC and for associations with clinical outcomes based upon stage of disease. We identified 22 miRNAs that were most differentially expressed in the plasma of healthy controls and all CRC patients or early stage (stage I-II) patients; in early stage and late stage (stage III-IV) patients; in stage I-III and patients with metastatic disease (stage IV); and in stage II and III CRC patients for which prognosis is important in decisions regarding post-operative adjuvant chemotherapy. In the clinical validation step, 11 miRNAs were examined in 234 plasma samples from CRC patients with follow-up data and from healthy controls. A comprehensive analysis revealed plasma miRNAs that may be useful for CRC prognosis after establishment of clinical utility.

\section{RESULTS}

\section{Study population}

The discovery set consisted of $40 \mathrm{CRC}$ patients (10 each of stage I-IV) and 10 healthy controls. All cases were age- and sex-matched, and no significant differences were seen in clinical parameters between the selected cases and the remaining patients in each comparison group (Table 1). Two hundred thirty-four cases, including 47 healthy controls and 187 CRC patients, were used to validate selected miRNAs. Their characters were shown in Table 1.

\section{Identification of candidate miRNAs in discovery study}

Using the TaqMan ${ }^{\circledR}$ Array Human MicroRNA A + B Cards Set v3.0, we profiled the levels of 754 miRNAs in 50 plasma samples from 10 healthy controls and 40 CRC patients (10 each of stage I-IV). The raw data and processed data were summarized in Table S2. In the analysis, we first determined the normalization reference, because no standard reference miRNA has been established for the studies of circulating miRNAs [24]. Although the TaqMan array provides U6, RNU44, and RNU48 as internal controls, which are often used in the normalization for cellular miRNAs, the levels of RNU44 and RNU48 were too low across all samples to be useful (Figure S1A). We therefore calculated the standard deviations of expression of all the miRNAs and found that six in card A (miR16, miR-17, miR-103, miR192, miR-451, and miR-93) and six in card B (miR-877, miR-188, miR-138-1, miR-520c-3p, miR-610, and U6) had relatively high RNA yields and the least expression variation across the discovery cohort (Figure S1B and S1C). Further analyses showed that the expression levels of miR-451 and miR-877 were not significantly different between healthy controls and CRC patients (Table S3) and were not significantly associated with tumor stage, and were therefore chosen as the normalization references to quantify plasma miRNAs in cards A and B, respectively.

We performed several pairwise comparisons in the individual 50 samples and identified 22 miRNAs with differential expression levels between groups (Figure 2, Table S4). Among the seven miRNAs that exhibited different expression levels between CRC patients and controls, let-7f-2* $(P=0.008)$, miR-15b* $(P<0.001)$, miR-526b $(P<0.001)$, miR-628-5p $(P<0.001)$, and miR486-3p $(P<0.001)$ had higher levels in CRC patients, respectively, while miR-801 $(P<0.001)$ and miR-376c $(P<0.001)$ had higher levels in controls. Six miRNAs had distinctive expression in early-stage CRC, including higher level of miR-96 $(P=0.031)$ and lower levels of miR-30a-5p $(P=0.037)$, miR-766 $(P=0.027)$, miR$197(P=0.039)$, miR-148a $(P<0.001)$, and miR-130b $(P=0.002)$, respectively. In advanced stage CRC, miR$203(P=0.035)$ and miR-200b $(P=0.005)$ were relatively enriched, whereas miR-22 $(P=0.003)$ was lower. Five miRNAs, including miR-31 $(P<0.001)$, miR-191 $(P=0.006)$, miR-155 $(P<0.002)$, miR-126 $(P=0.023)$ and miR-141 $(P=0.056)$, were markedly higher or showed a trend of being higher in stage IV CRC, while miR-519b-3p had lower levels $(P=0.003)$ in stage IV CRC. In addition, we found that miR-96 levels were higher in stage II than in stage III $(P=0.024)$.

\section{Clinical validation of candidate miRNAs in a second large cohort of CRC patients and controls}

We selected the 11 miRNAs for clinical validation in a larger cohort (187 CRC cases and 47 healthy controls) using quantitative RT-PCR and spiked-in cel-miR-39 as a normalizer. We first validated miR-141 since it was indicated to be a biomarker for metastatic CRC in our previous study [11]. Consistently, plasma miR-141 levels were higher in stage IV CRC patients than in stage I-III $(P<0.001$, Figure S2A). A further detailed analysis 
at each stage showed that the plasma miR-141 levels in stage IV CRC patients were significantly higher than in controls and stage I, stage II, and stage III CRC patients, respectively $(P<0.001$ for all, Figure S2B). In addition, CRC patients of all stages had higher miR-141 levels than did controls $(P<0.001$, Figure S2C), and miR-141 levels of stage III-IV patients were higher than those of controls and stage I-II patients $(P<0.001$, Figure S2D). The detailed data were also seen in Table S5.

Screening phase $(n=50)$

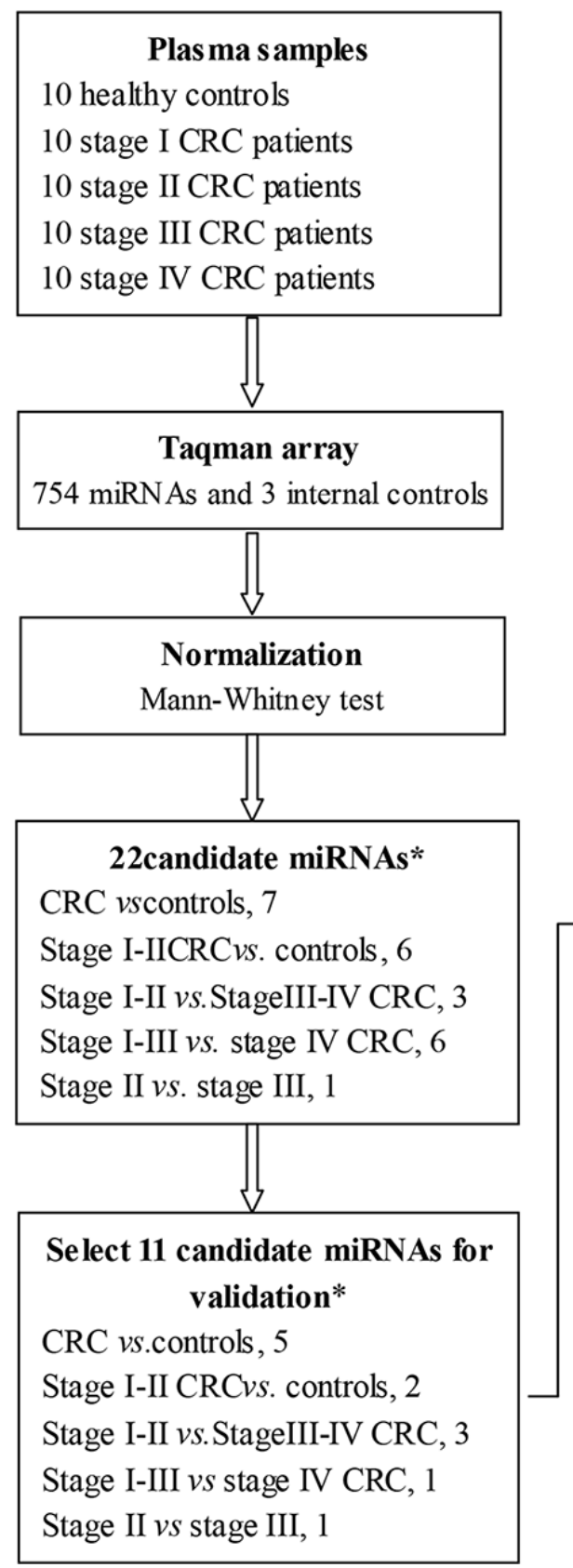

We compared the levels of plasma let-7f-2*, miR-15b*, miR-526b, miR-628-5p, and miR486-3p between CRC patients and controls which were suggested to be different in the discovery phase, and demonstrated the significant difference of let-7f-2* $(P=0.019$, Figure $3 \mathrm{~A})$ and miR$628-5$ p $(P=0.037$, Figure $3 \mathrm{E})$ levels in the validation cohort (Table S5). A detailed analysis further revealed that only stage IV CRC patients had significantly higher let-7f-2* $(P=0.001$, Figure 3B $)$ and miR-628-5p

\section{Validation phase $(n=234)$}

\begin{tabular}{|l|}
\hline \multicolumn{1}{|c|}{ Plas ma samples } \\
47 healthy controls \\
38 stage I CRC patients \\
50 stage II CRC patients \\
50 stage III CRC patients \\
49 stage IV CRC patients \\
\hline
\end{tabular}
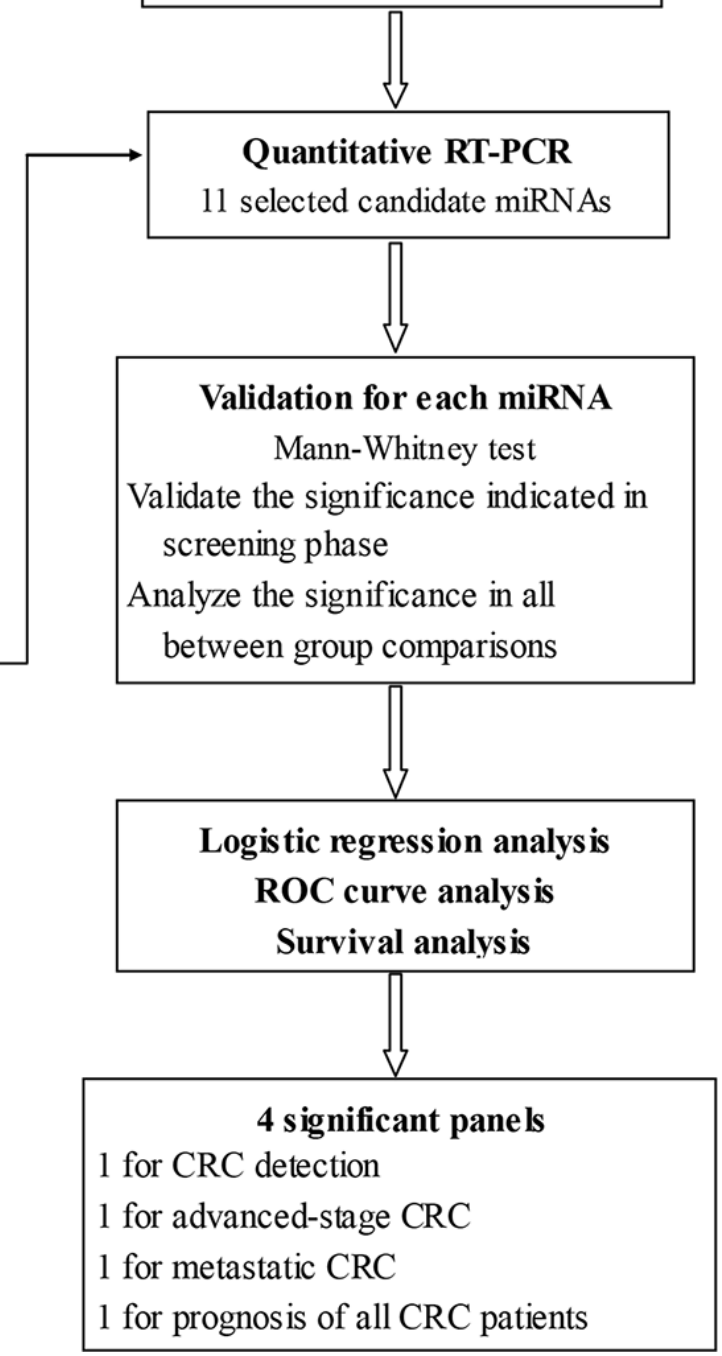

Figure 1: Study design. The microRNA (miRNA) profiles of 284 plasma samples from 227 CRC patients and 57 healthy controls were used to generate outcomes in 2 different phases. The candidate miRNAs discovered on 50 plasma samples using Taqman arrays were validated in 234 plasma samples using quantitative RT-PCR. The logistic regression, ROC curve and survival analyses were performed in the validation cohort. CRC: colorectal cancer. ${ }^{*} 1$ miRNAs repeated to be candidates in different comparisons. 
Table 1: Characteristics of healthy controls and colorectal cancer patients in this study

\begin{tabular}{|c|c|c|c|}
\hline Variable & Screening cohort & Validation cohort & $P$ value \\
\hline Healthy control, $n$ & 10 & 47 & \\
\hline Sex, $n(\%)$ & & & 0.854 \\
\hline Male & $5(50)$ & $22(46.8)$ & \\
\hline Female & $5(50)$ & $25(53.2)$ & \\
\hline Age $($ mean \pm SD) & $54 \pm 6.4$ & $54 \pm 6.3$ & 0.913 \\
\hline CRC patients, $n$ & 40 & 187 & \\
\hline Sex, $n(\%)$ & & & 0.735 \\
\hline Male & $20(50)$ & $99(52.9)$ & \\
\hline Female & $20(50)$ & $88(47.1)$ & \\
\hline Age $($ mean \pm SD $)$ & $55 \pm 6.3$ & $55 \pm 7.8$ & 0.773 \\
\hline Stage, $n(\%)$ & & & 0.933 \\
\hline $\mathrm{I}$ & $10(25)$ & $38(20.3)$ & \\
\hline II & $10(25)$ & $50(26.7)$ & \\
\hline III & $10(25)$ & $50(26.7)$ & \\
\hline IV & $10(25)$ & $49(26.2)$ & \\
\hline Surgery, $n(\%)$ & & & 0.887 \\
\hline Yes & $33(82.5)$ & $156(83.4)$ & \\
\hline No & $7(17.5)$ & $31(16.6)$ & \\
\hline Chemotherapy*, $n(\%)$ & & & 0.898 \\
\hline Yes & $31(77.5)$ & $144(77.0)$ & \\
\hline No & $9(22.5)$ & $42(22.5)$ & \\
\hline Status, $n(\%)$ & & & 0.420 \\
\hline Living & $31(77.5)$ & $128(68.4)$ & \\
\hline Dead & $8(20.0)$ & $46(24.6)$ & \\
\hline No information & $1(2.5)$ & $13(7.0)$ & \\
\hline
\end{tabular}

*No chemotherapy information was available for one patient.

$(P=0.001$, Figure 3F) levels than did controls (Table S5). Moreover, the CRC patients in stage IV had higher let-7f-2* and miR-628-5p levels than those with stage I-III CRC $(P=0.010$, Figure $3 \mathrm{C} ; P=0.010$, Figure $3 \mathrm{G})$. In addition, both let-7f-2* and miR-628-5p levels were significantly higher in stage III-IV than those in stage I-II CRC patients $(P=0.026$, Figure 3D; $P=0.006$, Figure $3 \mathrm{H})$. Although there was no significant difference in plasma miR-15b*, miR486-3p, and miR-526b levels between controls and CRC patients, miR-15b* and miR-526 levels were significantly higher in stage IV CRC patients than in controls (Table S5).

We performed the validation of miR-96 and miR-148a as candidates of early-stage CRC markers. Consistent with the discovery results, miR-96 levels were significantly higher in stage I-II CRC than in controls $(P=0.003$, Figure 4A). Plasma miR-96 levels in stage III-IV CRC patients were also higher than those in controls $(P=0.007$, Figure 4A). Therefore, all CRC patients had higher miR-96 levels than did controls $(P=0.003$, Figure 4B). A further analysis showed that compared with controls, miR-96 levels were higher in stage I $(P=0.019)$, stage II $(P=0.009)$, and stage IV CRC patients $(P<0.001)$. Interestingly, plasma miR-96 decreased from stage II to stage III $(P=0.043)$, and then showed the highest levels in stage IV (Figure 4C). CRC patients in stage IV had higher plasma miR-96 levels than stage I-III patients $(P=0.001$, Figure 4D). Inconsistent with the discovery data, plasma miR-148a showed the trend of having higher levels in stage I-II CRC than in controls in validation cohort $(P=0.064$, Figure 4E). Furthermore, miR-148a levels in stage III-IV CRC patients were higher than in controls $(P=0.001$, Figure 4E). Altogether, miR-148a levels were higher in all CRC patients than in controls $(P=0.003$, Figure $4 \mathrm{~F})$. In each group, miR-148a levels were significantly higher in stage II $(P=0.041)$, stage III $(P=0.037)$, and stage IV CRC patients $(P<0.001)$ than in controls (Figure $4 \mathrm{G})$. In addition, miR-148a levels were higher in stage IV patients than in stage I-III $(P=0.006$, Figure $4 \mathrm{H})$. The detailed data were also seen in Table S5. 
Consistent with the discovery results, plasma miR203 and miR-200b levels were significantly higher in stage III-IV CRC patients than in controls $(P<0.001$ for both) and stage I-II CRC patients $(P=0.006$ and $P<0.001$, respectively) in the validation cohort (Figure 5A, 5E). CRC patients had higher miR-203 and miR-200b levels than did controls $(P=0.003$, Figure $5 \mathrm{~B}$; $P=0.012$, Figure 5F). Plasma miR-203 and miR-200b levels were significantly higher in stage IV CRC patients, not in stage III, than in controls and each stage CRC patients (Figure 5C,5G). Altogether, stage IV CRC patients had higher plasma miR-203 and miR-200b levels than the patients of stage I-III $(P<0.001$ for both, Figure $5 \mathrm{D}, 5 \mathrm{H})$. In contrast to the result form discovery phase, plasma miR-22 levels were higher in stage III-IV CRC patients than in controls in the validation cohort $(P=0.009$, Figure 5I). They were also significantly higher in CRC patients than in controls $(P=0.019$, Figure 5J). Compared with controls, both stage III and IV CRC patients had higher miR-22 levels $(P=0.027, P=0.016$, respectively; Figure 5K).
Same with the results of the discovery phase, miR-96 levels were significantly higher in stage II than stage III CRC patients in the validation cohort $(P=0.043$, Figure 4C).

Plasma miR-451 levels were also validated in the validation cohort to determine whether it was stable across all samples. The results showed that there was no significant difference in miR-451 levels between each group $(P>0.05$ for all, Figure S3). In addition, we performed the same analysis for the 11 miRNAs using miR-451 as an endogenous control and found that the results were similar to those using cel-miR-39 as a control (Table S6).

\section{Potential microRNAs for CRC detection and stage}

We performed a combined analysis with each candidate miRNA in the validation set. The above results from the validation cohort showed that plasma levels of let-7f-2*, miR-628, miR-96, miR-148a, miR-203, miR200b, miR-22 and miR-141 were significantly different

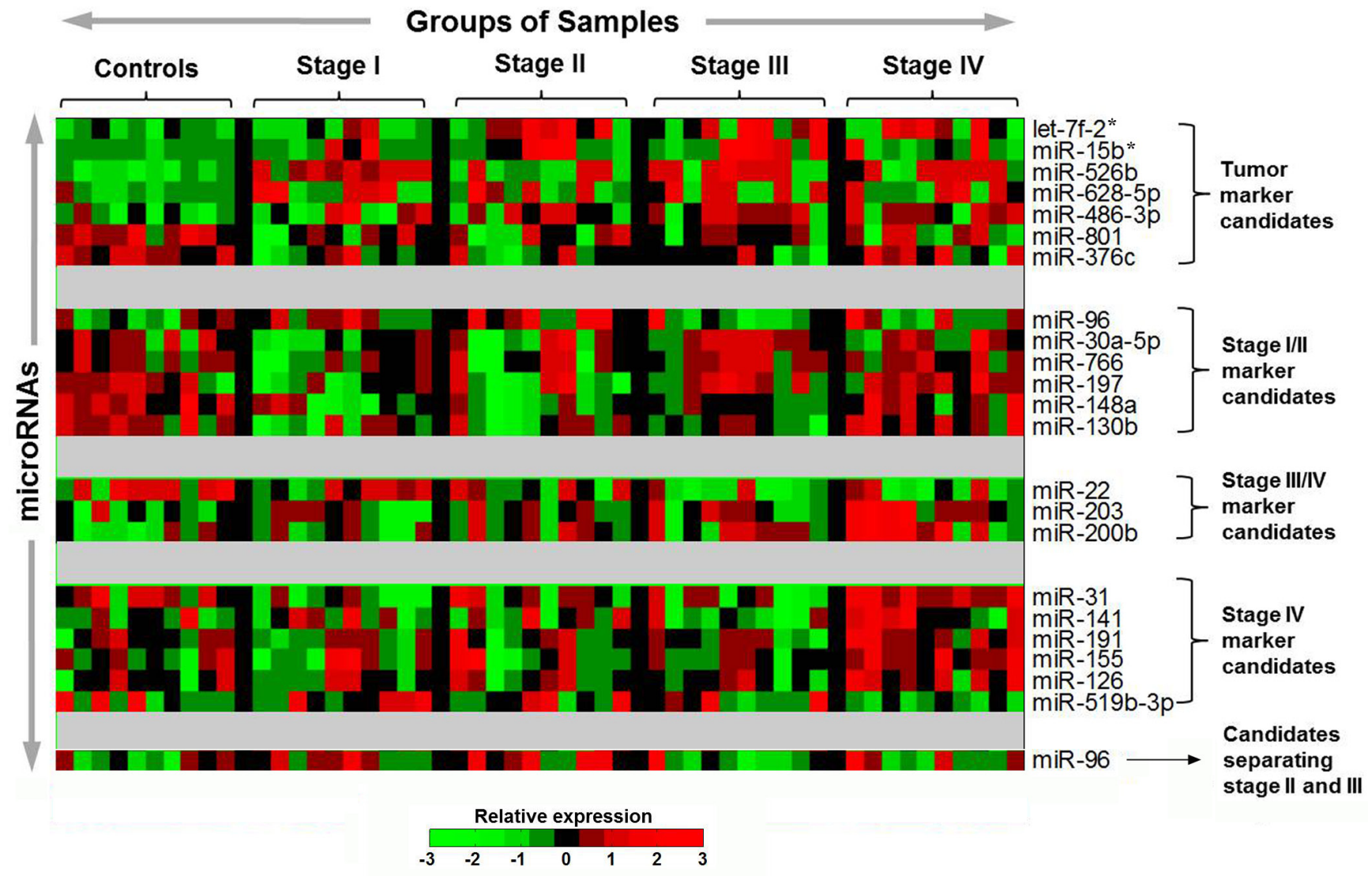

Figure 2: Potential plasma microRNA candidates selected from the TaqMan microarrays with 754 human miRNAs in the discovery study. The heatmap shows the relative expression pattern of the potential miRNA marker candidates across the 10 healthy controls and 40 CRC patients. The patient samples were grouped and ordered on the basis of tumor stage (10 samples per each of stage I-IV). Each row corresponds to plasma miRNA and each column corresponds to an individual sample. Expression levels of each miRNA are normalized across the samples such that the mean is zero and the standard deviation is equal to 1. Expression levels greater than the mean are shaded in red, and those below the mean are shaded in green. The miRNAs on the row were grouped on the basis of different biomarker categories. 

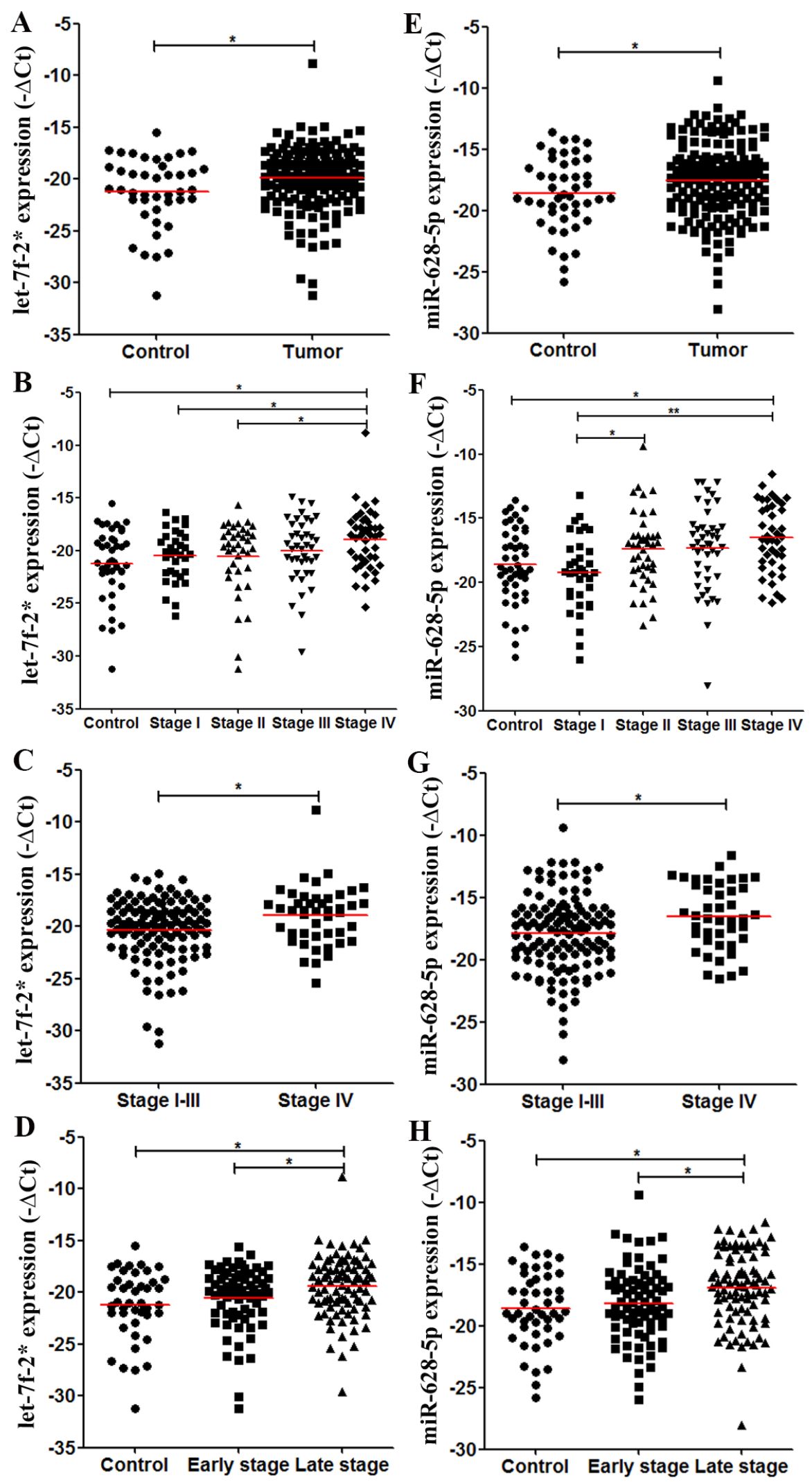

Figure 3: Levels of plasma miRNA candidates as potential CRC markers in the validation cohort. The levels of plasma miRNAs from 187 CRC patients and 47 healthy controls were examined using real-time RT-PCR and normalized with cel-miR-39 as the control. (A-D) Levels of plasma let-7f-2* in healthy controls and all CRC patients (A); in controls and each stage of CRC patients (B); in stage I-III and stage IV CRC patients (C); and in controls, stage I-II and stage III-IV CRC patients (D). (E-H) Levels of plasma miR-628-5p in multiple comparisons same with (A-D). ${ }^{*} P<0.05 ; * * P<0.01$. Exact $p$-values for each comparison were listed in Table S5. 

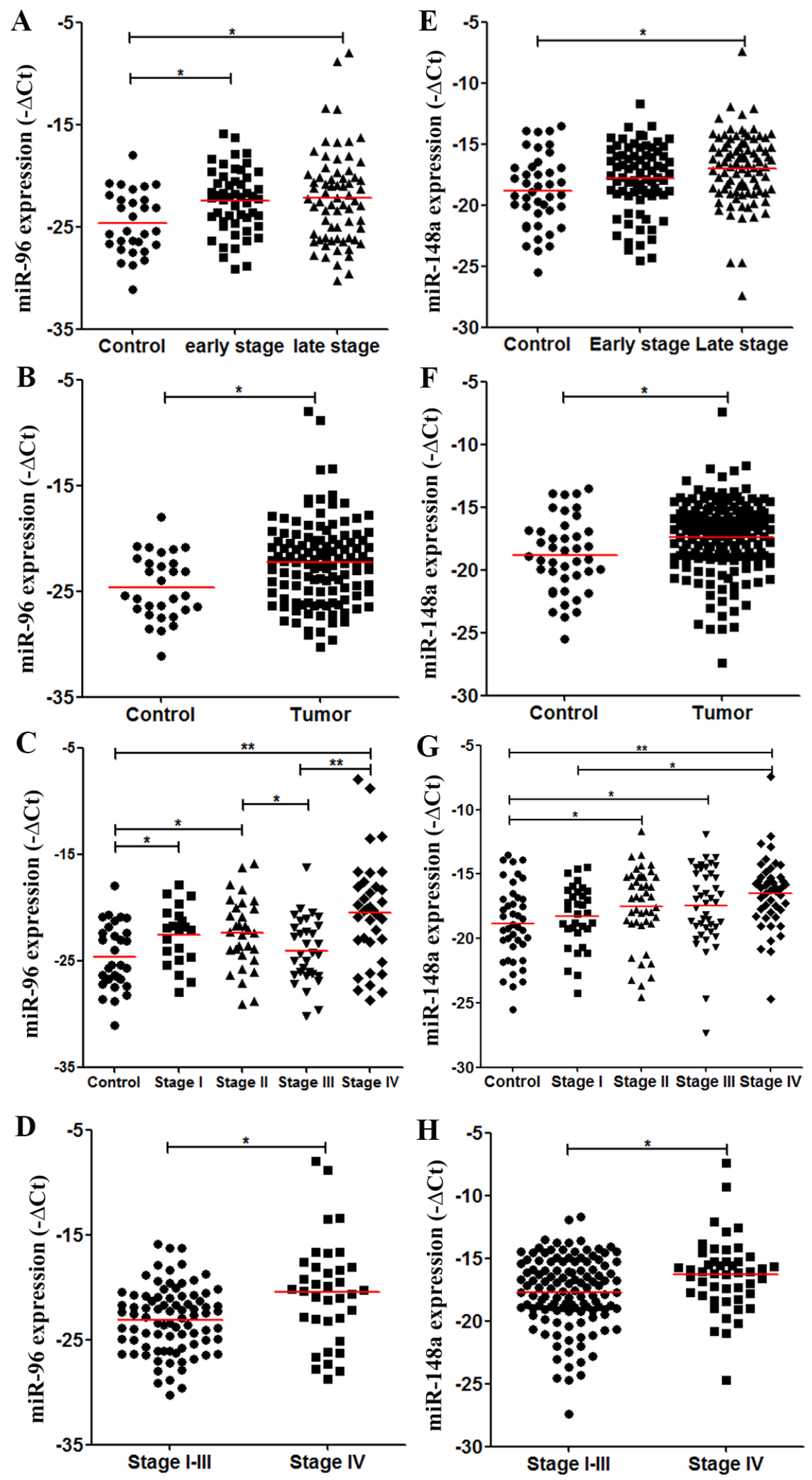

Figure 4: Levels of plasma miRNA candidates as potential early-stage CRC markers in the validation cohort. (A-D) Levels of plasma miR-96 in healthy controls, stage I-II and stage III-IV CRC patients (A), in healthy controls and all CRC patients (B), in healthy controls and each stage CRC patients (C), and in stage I-III and stage IV CRC patients (D). (E-H) Levels of plasma miR-148a in multiple comparisons same with (A-D). ${ }^{*} P<0.05 ; * * P<0.01$. Exact $p$-values for each comparison were listed in Table S5. 

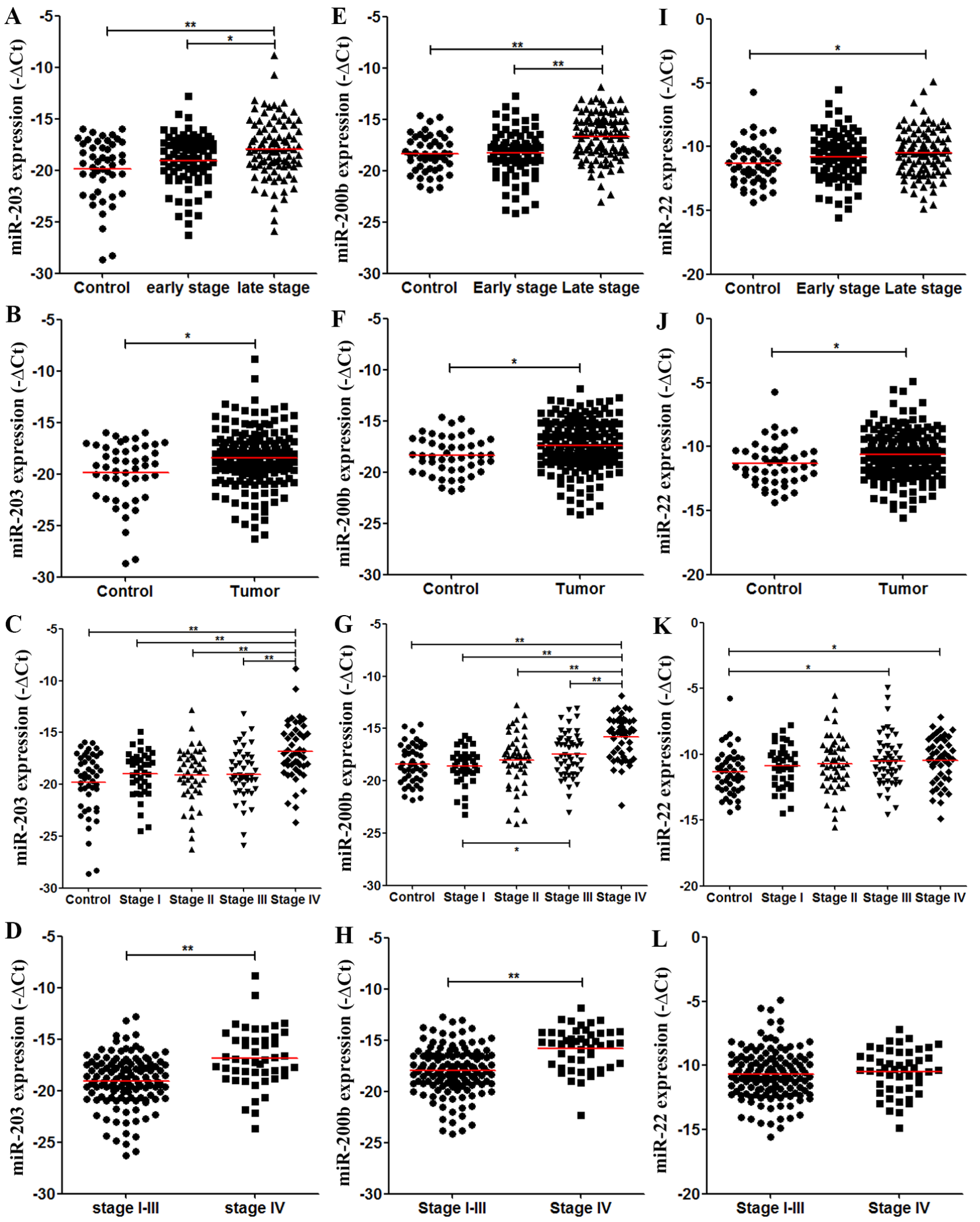

Figure 5: Levels of plasma miRNA candidates as potential late-stage CRC markers in the validation cohort. (A-D) Levels of plasma miR-203 in healthy controls, stage I-II and stage III-IV CRC patients (A), in healthy controls and all CRC patients (B), in controls and each stage CRC patients (C), and in stage I-III stage and IV CRC patients (D). (E-H) Levels of plasma miR-200 b in multiple comparisons same with (A-D). (I-L) Levels of plasma miR-22 in multiple comparisons same with (A-D). ${ }^{*} P<0.05 ; * * P<0.01$. Exact $p$-values for each comparison were listed in Table S5. 
between all CRC patients and controls. A logistic regression analysis identified the best model based on miR-96, $\operatorname{logit}(\mathrm{p})=12.918+0.149 *$ miR-96. At the optimal cut-off, the predicated probability based on plasma miR-96 levels had $65.4 \%$ sensitivity and $73.3 \%$ specificity in distinguishing between all CRC patients and controls, with an AUC of 0.740 [SE $=0.046,95 \%$ CI: $0.650-0.831]$ (Figure 6A). The validation data showed that the levels of let-7f-2*, miR-628-5p, miR-203, miR-200b and miR141 were significantly different between stage I-II and stage III-IV CRC patients. The best logistic model, logit (p) $=9.181+0.186^{*} \mathrm{miR}-203$, was established to distinguish between stage I-II and stage III-IV CRC patients. At the optimal cut-off, the predicated probability on the basis of plasma miR-203 had $74.7 \%$ sensitivity and $71.4 \%$ specificity, with an AUC of 0.757 [SE $=0.041,95 \%$ CI: 0.676-0.838] (Figure 6B). According to the results from the validation cohort, plasma let-7f-2*, miR-628-5p, miR-96, miR-148a, miR-203, miR-200b, and miR-141 levels were significantly different between stage I-III and stage IV CRC patients. A logistic regression analysis demonstrated the best logistic model based on miR-141, $\operatorname{logit}(\mathrm{p})=13.888+0.395 * \mathrm{miR}-141$. At the optimal cut-off, the model had $80.0 \%$ sensitivity and a $86.1 \%$ specificity for distinguishing between stage IV and stage I-III CRC patients, with an AUC of 0.851 [SE $=0.046,95 \% \mathrm{CI}$ : 0.762-0.940] (Figure 6C).

\section{Survival analyses for CRC patients}

We performed univariate survival analysis for the 11 candidate miRNAs identified from screening data in the validation cohort. Plasma miR-96, miR-200b and miR-141 were demonstrated to be associated with overall survival in CRC patients using a univariate survival analysis (Figure 7A-7C). The patients with lower levels of plasma miR-96 $(P=0.002)$, miR-200b $(P<0.001)$, and
miR-141 ( $P=0.005)$ showed better survival rate than those with higher levels of the corresponding miRNA. The multiple survival analysis, including known clinical parameters, serum CEA, and the three plasma miRNAs, revealed that stage, serum CEA, plasma miR-200b $(P=0.008$, risk ratio $(\mathrm{RR})=2.630)$, and miR-96 $(P=0.019$, $\mathrm{RR}=2.275)$ were independent factors for overall survival of CRC patients (Table 2).

\section{DISCUSSION}

In our two-tiered study, parts of the results from the discovery phase were clinically validated in an independent large-scale cohort. Our results suggest that the high throughput method of miRNA profiling in a sufficient number of plasma samples could identify candidate biomarkers. The logistic regression, ROC curve, and survival analyses identified the most significant miRNAs for potential clinical use, and the detailed analysis in healthy controls and CRC patients with each stage supplied candidates (miR-96, miR-203, miR-141 and miR-200b) for prognosis of CRC patients if clinical utility studies are completed successfully. The proposed functions and expression of the four candidate miRNAs in CRC tissue and circulation from literature [11, 18, 22, 25-49] were summarized in Table S7.

miR-96, a member of the miR-183 family, has been identified as a potential oncogene in several tumor types [26, 50-55]. Several studies showed that miR-96 was upregulated in CRC tissues compared to in normal mucosal tissue [25-31], and miR-96 in CRC tissue was found to be associated with liver metastasis [29]. These results in CRC tissue are consistent with our data about miR-96 in plasma. In the current study, plasma miR-96 exhibited significantly higher levels in all CRC patients than healthy controls. Plasma miR-96 levels in stage IV CRC patients were significantly higher than those in
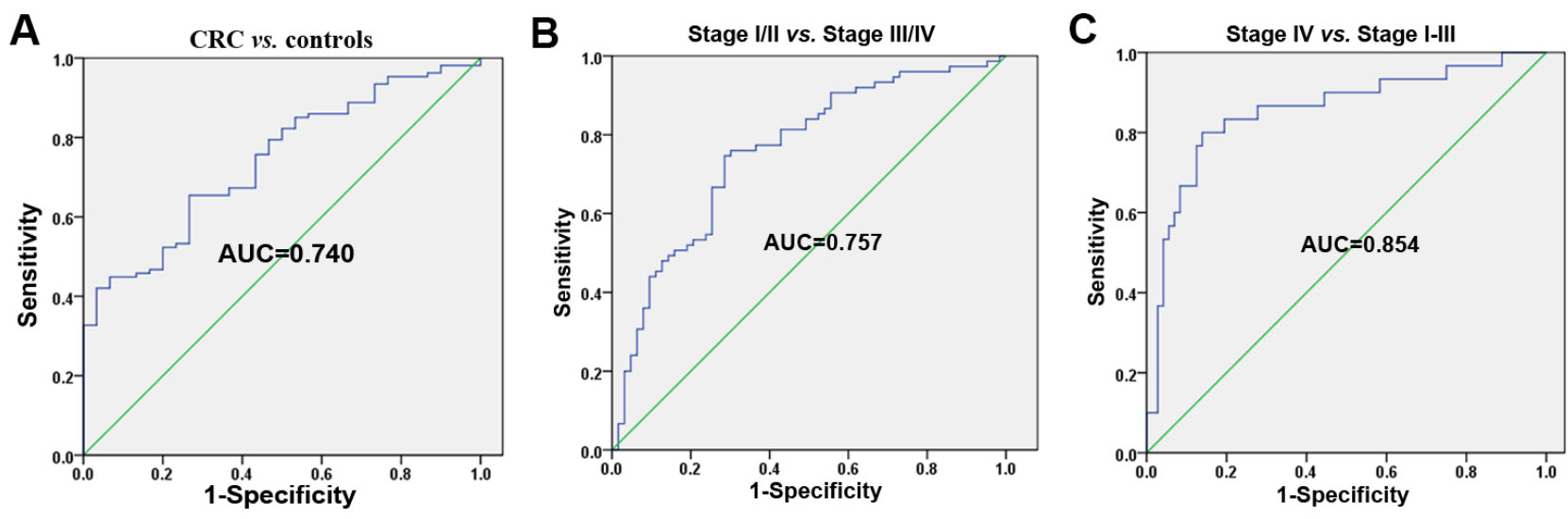

Figure 6: Integrated analyses of the potential microRNAs for CRC detection and stage in the validation cohort. (A) ROC analysis of the logistic regression model based on plasma miR-96 for separating CRC patients $(n=187)$ from healthy controls $(n=47)$. (B) ROC analysis of the logistic regression model based on plasma miR-203 for differentiating stage III-IV ( $n=99)$ from stage I-II CRC patients $(n=88)$. (C) ROC analysis of the logistic regression model based on plasma miR-141 for separating stage IV $(n=49)$ from stage I-III CRC patients $(n=138)$. 
stage I-III CRC patients. Moreover, plasma miR-96 level was suggested to be an independent prognostic marker for CRC patients. Brunet Vega et al. examined 11 miRNAs in the sera of 30 stage III CRC patients and 26 healthy controls and found that the mean fold change in serum miR-96 levels was 2.267, although the difference was not significant [30]. We obtained similar results in our study. Plasma miR-96 levels in stage I, II and IV CRC patients were significantly higher than those in healthy controls, but there was only a trend that plasma miR-96 levels in stage III CRC patients were higher than those in controls. Although we do not know the reason why plasma miR-96 levels decreased from stage II to stage III, the difference of miR-96 levels between stage II and stage III was identified in the screening phase and validated in the validation cohort. Considering the lymph node metastasis cannot be identified accurately by iconography before surgery, plasma miR-96 level may potentially contributes to distinguishing stage II and stage III CRC, which will help oncologist choose suitable treatment for these CRC patients. Therefore, plasma miR-96 is a potentially useful clinical marker in CRC prognosis and treatment, especially in stage II and III CRC patients.

The biological role of miR-203 is heterogeneous. miR-203 has been reported to function as a tumor suppressor in head and neck squamous cell carcinomas, esophageal squamous cell carcinoma, and cervical and prostate cancer [56-58]; miR-203 was suggested to be an oncogene in pancreatic, kidney and ovarian cancers [59-61]. The role of miR-203 in CRC is also inconsistent [32, 34-39]. Bovell et al. found higher expression of miR-203 in CRC than in corresponding normal tissues, and high miR-203 expression was associated with poor survival in white patients with stage IV CRC and black patients with stage I and II CRC [34]. Four other studies also reported that miR-203 in CRC tissue was higher than normal tissue [35-38]. However, two studies showed that miR-203 had a decreased expression in CRC tissue $[32,39]$, and the miR-203 downregulation was correlated with tumor size and pT stage [39]. Wang et al. reported that serum miR-203 levels were lower in CRC patients than in healthy controls [22]. In our study, plasma miR-203 levels were significantly higher in all CRC patients than in controls, and they were higher in stage III-IV than stage I-II CRC patients. The discrepancy across the different studies might be partly due to the difference in sample origin (serum or plasma), patient numbers, technology platforms, and the endogenous controls for normalization. Furthermore, circulating miR-203 appears to be a highly dynamic miRNA that changes under different physiological conditions. Further studies are needed to evaluate the role of circulating miR-203 in CRC.

miR-141 and miR-200b belong to the miR-200 family that is known to be a regulator of epithelial-tomesenchymal transition (EMT) [41, 48]. Although miR-141 was demonstrated to inhibit CRC cell migration and invasion in vitro [43], significantly upregulated miR-141 expression was observed in the plasma of metastatic CRC patients [11, 45]. Consistent with our previous study [11], the present study demonstrated that plasma miR-141 was a potential biomarker for metastatic CRC again. Recent studies have revealed complex functions associated with miR-200 family members [48]. The EMT inhibitor miR-200b was shown to stimulate tumor growth in TGFBR2-null CRC by targeting CDKN1B and negatively regulating p27/kip1 [46]. Toiyama et al. didn't find the significant difference of serum miR-200b levels between stage I and stage IV patients in their identification phase. However, they showed that serum miR-200c levels were significantly higher levels in stage IV than stage I-III CRC patients, and high serum miR-200c levels were positively correlated with lymph node metastasis, distant metastasis, and prognosis and were an independent prognostic marker for CRC [18]. In our current study, we observed that plasma miR-200b levels were higher in stage III-IV $\mathrm{CRC}$, especially in metastatic $\mathrm{CRC}$, and emerged as an independent prognostic marker for $\mathrm{CRC}$ patients.

We did not confirm most circulating miRNAs that were reported previously. One of the reasons may be that we performed many comparisons between different
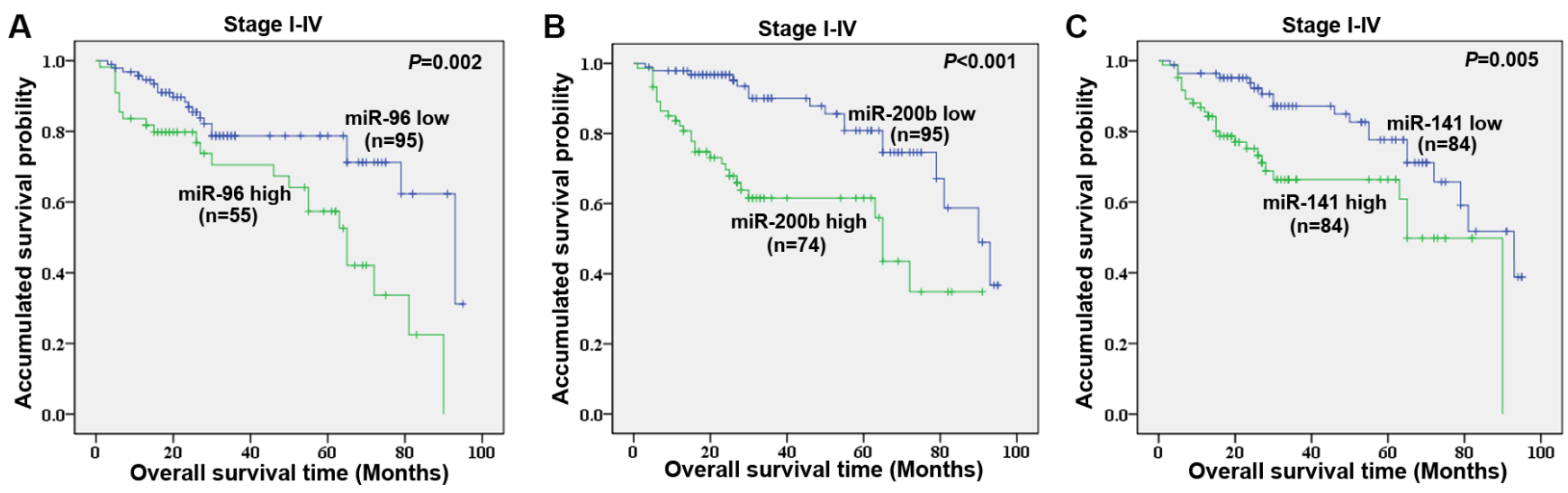

Figure 7: Univariate survival analyses of plasma miR-96 (A), miR-200b (B), and miR-141 (C) levels in stage I-IV CRC patients in the validation cohort using the Kaplan-Meier method and log-rank test. 
Table 2: Multivariate analyses of overall survival in $187 \mathrm{CRC}$ patients

\begin{tabular}{|l|c|c|c|}
\hline \multicolumn{1}{c|}{ Factor } & P value & Risk ratio & 95\% CI \\
\hline Stage (III-IV $v s$ I-II) & 0.001 & 4.746 & $1.917-11.751$ \\
\hline CEA $(>5 \mu \mathrm{g} / 1$ vs $\leq 5 \mu \mathrm{g} / \mathrm{l})$ & 0.001 & 3.412 & $1.612-7.219$ \\
\hline miR-200b (high $v s$ low) & 0.008 & 2.630 & $1.287-5.375$ \\
\hline miR-96 (high $v s$ low) & 0.019 & 2.275 & $1.147-4.512$ \\
\hline Sex (female $v s$ male) & 0.307 & 0.683 & $0.328-1.420$ \\
\hline miR-141 (high $v s$ low) & 0.335 & 1.555 & $0.778-3.106$ \\
\hline Chemotherapy (yes $v s$ no) & 0.396 & 0.684 & $0.284-1.646$ \\
\hline Age $(\geq 55$ years $v s<55$ years) & 0.575 & 1.232 & $0.594-2.552$ \\
\hline
\end{tabular}

groups and chose relatively fewer miRNAs as candidates based on each comparison in the discovery phase, so that some miRNAs with relatively lower significance were not selected in our study. However, the inconsistency of the results from multiple studies on circulating miRNAs has been reported previously and clearly impedes their clinical usage $[62,63]$. The lack of reproducibility could be attributed to factors such as sample size, sample preparation, screening method, and data normalization and analysis. Data normalization has been a troubling factor because no standard reference miRNA has been established [24]. U6 was demonstrated not to be suitable as endogenous control for the quantification of circulating microRNAs [64]. Although miR-16 was used as an internal control in some studies of miRNAs $[9,15]$, the circulating levels of miR-16 were demonstrated to be associated with hemolysis and bowel preparation [65]. In addition, circulating miR-16 was suggested to be a potential biomarker in gastric, breast and hepatocellular cancer [66-68], suggesting it is not a stable internal control for blood samples. Our results showed that plasma levels of miR-451 were more stable among healthy controls and CRC patients with each stage. miR-451 was recommended as an internal control in other study [69]. In addition, the validation results for selected candidate miRNAs using spiked-in cel-miR-39 or miR-451 as internal control were consistent in this study. The data suggest that miR-451 serves as a stable control miRNA for circulating miRNA analyses, although it must be further validated to establish a standardized protocol for clinical use.

Although the plasma levels of let-7f-2*, miR-628, miR-96, miR-148a, miR-203, miR-200b, miR-22 and miR-141 were significantly different between CRC patients and controls in our validation phase, detailed analyses of each stage showed that the difference between CRC patients and controls might result from the remarkable difference between advanced stage CRC and controls. Margue et al. also found that the levels of cell-free miRNAs only change significantly at later stages of melanoma progression [70]. Thus, development of clinically useful circulating markers for cancer detection should consider both early and late stages of cancers.
In conclusion, on the basis of a microRNAome screening of 50 individual samples and clinical validation in a relatively large sample of 284 cases, we propose four miRNA candidates as non-invasive biomarkers for CRC prognosis. We believe these biomarkers have the potential to be useful in clinical settings and prospective studies of clinical utility are warranted. Of note, the robustness of these potential biomarkers on clinical benefit needs to be evaluated in the future by using an independent CRC sample cohort.

\section{MATERIALS AND METHODS}

\section{Patient characteristics and specimens}

This study was approved by The University of Texas MD Anderson Cancer Center (Houston, Texas) institutional review board. Plasma samples from healthy individuals and CRC patients (stages I-IV) were obtained from TexGen between 2002 and 2010, a collaboration of Texas Medical Center institutions that provides biological samples as well as epidemiological and clinical data [11]. Written informed consent had been obtained from all patients for use of specimens and clinical data. Blood samples from cancer patients had been obtained before colorectal surgical resection. Patients who had undergone preoperative radiotherapy or chemotherapy were excluded. Tumors were staged according to the American Joint Committee on Cancer TNM staging system for colorectal cancer [71]. To select samples in healthy individuals, we used asymptomatic and apparently healthy volunteers with no history of cancer. All volunteers were confirmed to be healthy, with no malignancy, by physical examination. The serum CEA levels and clinical data (including gender, age, race and stage) were acquired from the TexGen clinical database that had been developed from clinical records. Follow-up data on all CRC patients were acquired from TexGen clinical database, and the survival time was calculated from the date of diagnosis to the date of death or last follow-up in October 2010. The median follow-up time was 28 months. 


\section{RNA isolation from plasma}

Small RNA was enriched from all plasma samples using the mirVana PARIS RNA isolation kit (Ambion, Austin, TX). In brief, a $300 \mu \mathrm{L}$ aliquot of plasma was thawed on ice and centrifuged at $14,000 \mathrm{rpm}$ for 10 minutes to remove cells and cellular debris. Next, $250 \mu \mathrm{L}$ of supernatant was lysed with an equal volume of $2 x$ denaturing solution. For normalization of sampleto-sample variation during the RNA isolation procedures, 25 fmol of synthetic C. elegans miRNA (cel-miR-39, GE Dharmacon) was added to each denatured sample. Small RNAs were then enriched and purified following the manufacturer's protocol, with the exception that the enriched small RNAs were eluted in $20-30 \mu \mathrm{L}$ of preheated nuclease-free water. DNase (Qiagen) treatment was used to remove any contaminating DNA. The RNA concentration was quantified using NanoDrop1000 (NanoDrop, Wilmington, DE) in all samples and ranged from 3 to $35 \mathrm{ng} / \mu \mathrm{L}$.

\section{miRNA screening assay}

Complementary DNA (cDNA) was generated for each RNA sample from the plasma of the discovery set using reverse transcription with Megaplex RT primers, human pool set v3.0 (Life Technologies, Foster City, CA), which consists of 754 stem-looped reverse transcription primers plus three TaqMan-designated internal controls. The cDNA underwent pre-amplification with Megaplex PreAmp Primers, human pool set v3.0 (Life Technologies), and was loaded onto the TaqMan array human microRNA A + B cards set v3.0 (Life Technologies). These cards were run using the Applied Biosystems 7900HT real-time PCR system, according to the manufacturer's instructions.

Although the TaqMan array provides three internal controls (U6, RNU44, and RNU48) that are commonly used to measure cellular miRNAs, there are no standard endogenous control miRNAs, especially for an analysis of circulating miRNAs [24]. We first preprocessed the miRNAs by filtering out those with the same raw cycle threshold $(\mathrm{Ct})$ value in all samples in the whole cohort, corresponding to a standard deviation of zero. We next chose a normalization reference from the preprocessed miRNAs that was based on the following three criteria [64, 72]: (1) Small expression variation, in terms of standard deviation across the whole discovery cohort; (2) High RNA yield or absorbance in terms of the raw $\mathrm{Ct}$ values; and (3) No statistically significant difference in miRNA expression among samples in different categories (i.e., healthy controls vs CRC patients, or among stage I, II, III and IV CRC patients). The normalization was performed by subtracting the raw $\mathrm{Ct}$ values of the reference miRNA from the $\mathrm{Ct}$ values of all the other miRNAs and was expressed as $\Delta \mathrm{Ct} . \Delta \Delta \mathrm{Ct}$ was then calculated by subtracting the average $\Delta \mathrm{Ct}$ of the control from $\Delta \mathrm{Ct}$ of all samples. The relative expression levels of miRNAs were then calculated utilizing the $-\Delta \Delta \mathrm{Ct}$ method. The analysis was performed by using the Matlab software. The raw and processed data for Card A and Card B were shown in Table S2.

To determine whether candidate miRNAs could serve as effective markers, we performed several pairwise comparisons (control vs CRC, control vs stages I-II, control $v s$ stage III-IV, stage I-II $v s$ stage III-IV, control $v s$ stage IV, stage I-III $v s$ stage IV, and stage II $v s$ stage III). miRNAs that were significantly differentially expressed in these comparisons were chosen as potential candidates for further investigation.

\section{miRNA clinical validation study}

Eleven miRNAs selected from the discovery phase were analyzed using real time RT-PCR assays as described previously [11]. Expression levels of miRNAs were quantified in duplicate and spiked-in cel-miR-39 was used as normalizers for plasma miRNA quantification. The mean cycle threshold of the replicated measurement of each miRNA was included in the analysis. The software defaults of the 7900 Sequence Detection System 2.3 (Applied Biosystems) were used to compute the relative change in RNA expression with the $\Delta \mathrm{Ct}$ method and $95 \%$ confidence intervals.

\section{Statistical analysis}

Standard statistical tests including Student's $t$-test and Mann-Whitney test were used to assess difference in miRNA levels between different groups. In the validation cohort, receiver operating characteristic (ROC) curves [73] were generated to assess the diagnostic accuracy of each parameter, and the sensitivity and specificity of the optimum cut-off point were defined as those values that maximized the area under the ROC curve (AUC). Multivariate logistic regression analysis was performed to find the best miRNA panel for CRC detection or stage [9]. The AUC was used as an accuracy index for evaluating the diagnostic performance of the selected microRNA panels. The associations between overall survival and plasma miRNAs were analyzed using the Kaplan-Meier method and the log-rank test [74]. For each miRNA, the patients were divided into two groups (low and high levels) based on the mean of the relative expression levels of the miRNA in plasma. A Cox proportional-hazards regression analysis was used to evaluate the independent prognostic factors. Statistical analysis was performed using the software packages such as Matlab (MathWorks, Inc., Natick, Massachusetts), SPSS version 16.0 (WPSS, Ltd., Surrey, United Kingdom) and GraphPad Prism 5.0 (GraphPad Software, Inc., California). All statistical tests were two-sided, and a $P$ value of less than 0.05 was considered significant. 


\section{ACKNOWLEDGMENTS AND FUNDING}

We thank Bethany Alicie for technical assistance and Ann Sutton in the Department of Scientific Publications for editing this manuscript. This work was partially supported by National Institutes of Health grant CA 150654 (WZ and SRH), MD Anderson's National Cancer Institute Cancer Center Support Grant (CA16672), and a grant from the National Foundation for Cancer Research. Y. Sun was supported by The A. Lavoy Moore Endowment Fund, two grants from the National Nature Science Foundation of China (81472263 and 81201651), and a grant from the Tianjin Municipal Science and Technology Commission (14JCYBJC27500). S.R. Hamilton holds the Frederick F. Becker Distinguished University Chair in Cancer Research from The University of Texas. Dr. Calin is an Alan M. Gewirtz Leukemia and Lymphoma Society Scholar. Work in Dr. Calin's laboratory is supported in part by the NIH/NCI grants 1UH2TR00943-01 and R01 CA182905-01, the Laura and John Arnold Foundation, the RGK Foundation, and the Estate of C. G. Johnson, Jr.

\section{CONFLICTS OF INTEREST}

The authors declare no conflicts of interest.

\section{REFERENCES}

1. Siegel R, Ma J, Zou Z, Jemal A. Cancer statistics, 2014. CA Cancer J Clin. 2014; 64:9-29.

2. Walsh JM, Terdiman JP. Colorectal cancer screening: scientific review. JAMA. 2003; 289:1288-1296.

3. Collins JF, Lieberman DA, Durbin TE, Weiss DG. Accuracy of screening for fecal occult blood on a single stool sample obtained by digital rectal examination: a comparison with recommended sampling practice. Ann Intern Med. 2005; 142:81-85.

4. Fletcher RH. Carcinoembryonic antigen. Ann Intern Med. 1986; 104:66-73.

5. Fabbri M, Croce CM, Calin GA. MicroRNAs. Cancer J. 2008; 14:1-6.

6. Calin GA, Croce CM. MicroRNA signatures in human cancers. Nat Rev Cancer. 2006; 6:857-866.

7. Liu G, Xue F, Zhang W. miR-506: a regulator of chemosensitivity through suppression of the RAD51-homologous recombination axis. Chin J Cancer. 2015; 34:44.

8. Ng EK, Chong WW, Jin H, Lam EK, Shin VY, Yu J, Poon TC, Ng SS, Sung JJ. Differential expression of microRNAs in plasma of patients with colorectal cancer: a potential marker for colorectal cancer screening. Gut. 2009; 58:1375-1381.

9. Huang Z, Huang D, Ni S, Peng Z, Sheng W, Du X. Plasma microRNAs are promising novel biomarkers for early detection of colorectal cancer. Int J Cancer. 2010; 127:118-126.
10. Pu XX, Huang GL, Guo HQ, Guo CC, Li H, Ye S, Ling S, Jiang L, Tian Y, Lin TY. Circulating miR-221 directly amplified from plasma is a potential diagnostic and prognostic marker of colorectal cancer and is correlated with p53 expression. J Gastroenterol Hepatol. 2010; 25:1674-1680.

11. Cheng H, Zhang L, Cogdell DE, Zheng H, Schetter AJ, Nykter M, Harris CC, Chen K, Hamilton SR, Zhang W. Circulating plasma MiR-141 is a novel biomarker for metastatic colon cancer and predicts poor prognosis. PLoS One. 2011; 6:e17745.

12. Ahmed FE, Amed NC, Vos PW, Bonnerup C, Atkins JN, Casey M, Nuovo GJ, Naziri W, Wiley JE, Allison RR. Diagnostic microRNA markers to screen for sporadic human colon cancer in blood. Cancer Genomics Proteomics. 2012; 9:179-192.

13. Kanaan Z, Rai SN, Eichenberger MR, Roberts H, Keskey B, Pan J, Galandiuk S. Plasma miR-21: a potential diagnostic marker of colorectal cancer. Ann Surg. 2012; 256:544-551.

14. Wang Q, Huang Z, Ni S, Xiao X, Xu Q, Wang L, Huang D, Tan C, Sheng W, Du X. Plasma miR-601 and miR-760 are novel biomarkers for the early detection of colorectal cancer. PLoS One. 2012; 7:e44398.

15. Giraldez MD, Lozano JJ, Ramirez G, Hijona E, Bujanda L, Castells A, Gironella M. Circulating microRNAs as biomarkers of colorectal cancer: results from a genomewide profiling and validation study. Clin Gastroenterol Hepatol. 2013; 11:681-688 e683.

16. Luo X, Stock C, Burwinkel B, Brenner H. Identification and evaluation of plasma microRNAs for early detection of colorectal cancer. PLoS One. 2013; 8:e62880.

17. Yong FL, Law CW, Wang CW. Potentiality of a triple microRNA classifier: miR-193a-3p, miR-23a and miR-338$5 \mathrm{p}$ for early detection of colorectal cancer. BMC Cancer. 2013; 13:280.

18. Toiyama Y, Hur K, Tanaka K, Inoue Y, Kusunoki M, Boland CR, Goel A. Serum miR-200c is a novel prognostic and metastasis-predictive biomarker in patients with colorectal cancer. Ann Surg. 2014; 259:735-743.

19. Kanaan Z, Roberts H, Eichenberger MR, Billeter A, Ocheretner G, Pan J, Rai SN, Jorden J, Williford A, Galandiuk S. A plasma microRNA panel for detection of colorectal adenomas: a step toward more precise screening for colorectal cancer. Ann Surg. 2013; 258:400-408.

20. Kjersem JB, Ikdahl T, Lingjaerde OC, Guren T, Tveit KM, Kure EH. Plasma microRNAs predicting clinical outcome in metastatic colorectal cancer patients receiving first-line oxaliplatin-based treatment. Mol Oncol. 2014; 8:59-67.

21. Shivapurkar N, Weiner LM, Marshall JL, Madhavan S, Deslattes Mays A, Juhl H, Wellstein A. Recurrence of early stage colon cancer predicted by expression pattern of circulating microRNAs. PLoS One. 2014; 9:e84686.

22. Wang J, Huang SK, Zhao M, Yang M, Zhong JL, Gu YY, Peng H, Che YQ, Huang CZ. Identification of a circulating microRNA signature for colorectal cancer detection. PLoS One. 2014; 9:e87451. 
23. Du M, Liu S, Gu D, Wang Q, Zhu L, Kang M, Shi D, Chu H, Tong N, Chen J, Adams TS, Zhang Z, Wang M. Clinical potential role of circulating microRNAs in early diagnosis of colorectal cancer patients. Carcinogenesis. 2014; 35:2723-2730.

24. Heneghan HM, Miller N, Kerin MJ. Systemic microRNAs: novel biomarkers for colorectal and other cancers? Gut. 2010; 59:1002-1004.

25. Gao F, Wang W. MicroRNA-96 promotes the proliferation of colorectal cancer cells and targets tumor protein p53 inducible nuclear protein 1, forkhead box protein O1 (FOXO1) and FOXO3a. Mol Med Rep. 2015; 11:1200-1206.

26. Bandres E, Cubedo E, Agirre X, Malumbres R, Zarate R, Ramirez N, Abajo A, Navarro A, Moreno I, Monzo M, García-Foncillas J. Identification by Real-time PCR of 13 mature microRNAs differentially expressed in colorectal cancer and non-tumoral tissues. Mol Cancer. 2006; 5:29.

27. Sarver AL, French AJ, Borralho PM, Thayanithy V, Oberg AL, Silverstein KA, Morlan BW, Riska SM, Boardman LA, Cunningham JM, Subramanian S, Wang L, Smyrk TC, et al. Human colon cancer profiles show differential microRNA expression depending on mismatch repair status and are characteristic of undifferentiated proliferative states. BMC Cancer. 2009; 9:401.

28. Hamfjord J, Stangeland AM, Hughes T, Skrede ML, Tveit KM, Ikdahl T, Kure EH. Differential expression of miRNAs in colorectal cancer: comparison of paired tumor tissue and adjacent normal mucosa using high-throughput sequencing. PLoS One. 2012; 7:e34150.

29. Xu XM, Qian JC, Deng ZL, Cai Z, Tang T, Wang P, Zhang KH, Cai JP. Expression of miR-21, miR-31, miR-96 and miR-135b is correlated with the clinical parameters of colorectal cancer. Oncol Lett. 2012; 4:339-345.

30. Brunet Vega A, Pericay C, Moya I, Ferrer A, Dotor E, Pisa A, Casalots A, Serra-Aracil X, Oliva JC, Ruiz A, Saigí E. microRNA expression profile in stage III colorectal cancer: circulating miR-18a and miR-29a as promising biomarkers. Oncol Rep. 2013; 30:320-326.

31. Kara M, Yumrutas O, Ozcan O, Celik OI, Bozgeyik E, Bozgeyik I, Tasdemir S. Differential expressions of cancerassociated genes and their regulatory miRNAs in colorectal carcinoma. Gene. 2015; 567:81-86.

32. Abella V, Valladares M, Rodriguez T, Haz M, Blanco M, Tarrio N, Iglesias P, Aparicio LA, Figueroa A. miR-203 regulates cell proliferation through its influence on Hakai expression. PLoS One. 2012; 7:e52568.

33. Ju SY, Chiou SH, Su Y. Maintenance of the stemness in CD44(+) HCT-15 and HCT-116 human colon cancer cells requires miR-203 suppression. Stem Cell Res. 2014; 12:86-100.

34. Bovell LC, Shanmugam C, Putcha BD, Katkoori VR, Zhang B, Bae S, Singh KP, Grizzle WE, Manne U. The prognostic value of microRNAs varies with patient race/ ethnicity and stage of colorectal cancer. Clin Cancer Res. 2013; 19:3955-3965.
35. Yantiss RK, Goodarzi M, Zhou XK, Rennert H, Pirog EC, Banner BF, Chen YT. Clinical, pathologic, and molecular features of early-onset colorectal carcinoma. Am J Surg Pathol. 2009; 33:572-582.

36. Schetter AJ, Leung SY, Sohn JJ, Zanetti KA, Bowman ED, Yanaihara N, Yuen ST, Chan TL, Kwong DL, Au GK, Liu CG, Calin GA, Croce CM, et al. MicroRNA expression profiles associated with prognosis and therapeutic outcome in colon adenocarcinoma. JAMA. 2008; 299:425-436.

37. Wu X, Li S, Xu X, Wu S, Chen R, Jiang Q, Li Y, Xu Y. The potential value of miR-1 and miR-374b as biomarkers for colorectal cancer. Int J Clin Exp Pathol. 2015;, 8:2840-2851.

38. Riordan AM, Thomas MK, Ronnekleiv-Kelly S, Warner T, Geiger PG, Kennedy GD. Utility of micro-ribonucleic acid profile for predicting recurrence of rectal cancer. J Surg Res. 2012,; 177:87-92.

39. Chiang Y, Song Y, Wang Z, Chen Y, Yue Z, Xu H, Xing C, Liu Z. Aberrant expression of miR-203 and its clinical significance in gastric and colorectal cancers. J Gastrointest Surg. 2011; 15:63-70.

40. Wu PP, Zhu HY, Sun XF, Chen LX, Zhou Q, Chen J. MicroRNA-141 regulates the tumour suppressor DLC1 in colorectal cancer. Neoplasma. 2015; 62:705-712.

41. Burk U, Schubert J, Wellner U, Schmalhofer O, Vincan E, Spaderna S, Brabletz T. A reciprocal repression between ZEB1 and members of the miR-200 family promotes EMT and invasion in cancer cells. EMBO Rep. 2008; 9:582-589.

42. Wellner U, Schubert J, Burk UC, Schmalhofer O, Zhu F, Sonntag A, Waldvogel B, Vannier C, Darling D, zur Hausen A, Brunton VG, Morton J, Sansom O, et al. The EMT-activator ZEB1 promotes tumorigenicity by repressing stemness-inhibiting microRNAs. Nat Cell Biol. 2009; 11:1487-1495.

43. Hu M, Xia M, Chen X, Lin Z, Xu Y, Ma Y, Su L. MicroRNA-141 regulates Smad interacting protein 1 (SIP1) and inhibits migration and invasion of colorectal cancer cells. Dig Dis Sci. 2010; 55:2365-2372.

44. Diaz T, Tejero R, Moreno I, Ferrer G, Cordeiro A, Artells R, Navarro A, Hernandez R, Tapia G, Monzo M. Role of miR-200 family members in survival of colorectal cancer patients treated with fluoropyrimidines. J Surg Oncol. 2014; 109:676-683.

45. Yin J, Bai Z, Song J, Yang Y, Wang J, Han W, Zhang J, Meng H, Ma X, Wang T, Li W, Zhang Z. Differential expression of serum miR-126, miR-141 and miR-21 as novel biomarkers for early detection of liver metastasis in colorectal cancer. Chin J Cancer Res. 2014; 26:95-103.

46. Fu Y, Liu X, Zhou N, Du L, Sun Y, Zhang X, Ge Y. MicroRNA-200b stimulates tumour growth in TGFBR2null colorectal cancers by negatively regulating p27/kip1. J Cell Physiol. 2014; 229:772-782.

47. Pan Y, Liang H, Chen W, Zhang H, Wang N, Wang F, Zhang S, Liu Y, Zhao C, Yan X, Zhang J, Zhang CY, Gu H, et al. microRNA-200b and microRNA-200c promote colorectal 
cancer cell proliferation via targeting the reversion-inducing cysteine-rich protein with Kazal motifs. RNA Biol. 2015; 12:276-289.

48. Tian Y, Pan Q, Shang Y, Zhu R, Ye J, Liu Y, Zhong X, Li S, He Y, Chen L, Zhao J, Chen W, Peng Z, et al. MicroRNA-200 (miR-200) cluster regulation by achaete scute-like 2 (Ascl2): impact on the epithelial-mesenchymal transition in colon cancer cells. J Biol Chem. 2014; 289:36101-36115.

49. Slattery ML, Herrick JS, Mullany LE, Valeri N, Stevens J, Caan BJ, Samowitz W, Wolff RK. An evaluation and replication of miRNAs with disease stage and colorectal cancer-specific mortality. Int J Cancer. 2015; 137:428-438.

50. Schaefer A, Jung M, Mollenkopf HJ, Wagner I, Stephan C, Jentzmik F, Miller K, Lein M, Kristiansen G, Jung K.: Diagnostic and prognostic implications of microRNA profiling in prostate carcinoma. Int $\mathrm{J}$ Cancer. 2010; 126:1166-1176.

51. Pineau P, Volinia S, McJunkin K, Marchio A, Battiston C, Terris B, Mazzaferro V, Lowe SW, Croce CM, Dejean A. miR-221 overexpression contributes to liver tumorigenesis. Proc Natl Acad Sci U S A. 2010; 107:264-269.

52. Sarver AL, Li L, Subramanian S. MicroRNA miR-183 functions as an oncogene by targeting the transcription factor EGR1 and promoting tumor cell migration. Cancer Res. 2010; 70:9570-9580.

53. Wang L, Zhu MJ, Ren AM, Wu HF, Han WM, Tan RY, Tu RQ. A ten-microRNA signature identified from a genome-wide microRNA expression profiling in human epithelial ovarian cancer. PLoS One. 2014; 9:e96472.

54. Li J, Li P, Chen T, Gao G, Chen X, Du Y, Zhang R, Yang R, Zhao W, Dun S, Gao F, Zhang G. Expression of microRNA-96 and its potential functions by targeting FOXO3 in non-small cell lung cancer. Tumour Biol. 2015; 36:685-692.

55. Li P, Sheng C, Huang L, Zhang H, Cheng Z, Zhu Q. MiR$183 /-96 /-182$ cluster is up-regulated in most breast cancers and increases cell proliferation and migration. Breast Cancer Res. 2014; 16:473.

56. Boldrup L, Coates PJ, Wahlgren M, Laurell G, Nylander K. Subsite-based alterations in miR-21, miR-125b, and miR203 in squamous cell carcinoma of the oral cavity and correlation to important target proteins. J Carcinog. 2012; 11:18.

57. Zhao S, Yao DS, Chen JY, Ding N. Aberrant expression of miR-20a and miR-203 in cervical cancer. Asian Pac J Cancer Prev. 2013; 14:2289-2293.

58. Xiang J, Bian C, Wang H, Huang S, Wu D. MiR-203 downregulates Rap1A and suppresses cell proliferation, adhesion and invasion in prostate cancer. J Exp Clin Cancer Res. 2015; 34:8.

59. Petillo D, Kort EJ, Anema J, Furge KA, Yang XJ, Teh BT. MicroRNA profiling of human kidney cancer subtypes. Int J Oncol. 2009; 35:109-114.
60. Ikenaga N, Ohuchida K, Mizumoto K, Yu J, Kayashima T, Sakai H, Fujita H, Nakata K, Tanaka M. MicroRNA-203 expression as a new prognostic marker of pancreatic adenocarcinoma. Ann Surg Oncol. 2010; 17:3120-3128.

61. Wang S, Zhao X, Wang J, Wen Y, Zhang L, Wang D, Chen H, Chen Q, Xiang W. Upregulation of microRNA-203 is associated with advanced tumor progression and poor prognosis in epithelial ovarian cancer. Med Oncol. 2013; 30:681.

62. Leidner RS, Li L, Thompson CL. Dampening enthusiasm for circulating microRNA in breast cancer. PLoS One. 2013; 8:e57841.

63. Faltejskova P, Bocanek O, Sachlova M, Svoboda M, Kiss I, Vyzula R, Slaby O. Circulating miR-17-3p, miR-29a, miR92a and miR-135b in serum: Evidence against their usage as biomarkers in colorectal cancer. Cancer Biomark. 2012; 12:199-204.

64. Xiang M, Zeng Y, Yang R, Xu H, Chen Z, Zhong J, Xie H, $\mathrm{Xu} \mathrm{Y}$, Zeng $\mathrm{X}$. U6 is not a suitable endogenous control for the quantification of circulating microRNAs. Biochem Biophys Res Commun. 2014; 454:210-214.

65. Yamada A, Cox MA, Gaffney KA, Moreland A, Boland CR, Goel A. Technical factors involved in the measurement of circulating microRNA biomarkers for the detection of colorectal neoplasia. PLoS One. 2014; 9:e112481.

66. Zhang J, Song Y, Zhang C, Zhi X, Fu H, Ma Y, Chen Y, Pan F, Wang K, Ni J, Jin W, He X, Su H, et al. Circulating MiR-16-5p and MiR-19b-3p as Two Novel Potential Biomarkers to Indicate Progression of Gastric Cancer. Theranostics. 2015; 5:733-745.

67. Stuckrath I, Rack B, Janni W, Jager B, Pantel K, Schwarzenbach H. Aberrant plasma levels of circulating miR-16, miR-107, miR-130a and miR-146a are associated with lymph node metastasis and receptor status of breast cancer patients. Oncotarget. 2015; 6:13387-13401. doi: 10.18632/oncotarget.3874.

68. El-Abd NE, Fawzy NA, El-Sheikh SM, Soliman ME. Circulating miRNA-122, miRNA-199a, and miRNA-16 as Biomarkers for Early Detection of Hepatocellular Carcinoma in Egyptian Patients with Chronic Hepatitis C Virus Infection. Mol Diagn Ther. 2015; 19:213-220.

69. Pritchard CC, Kroh E, Wood B, Arroyo JD, Dougherty KJ, Miyaji MM, Tait JF, Tewari M. Blood cell origin of circulating microRNAs: a cautionary note for cancer biomarker studies. Cancer Prev Res (Phila). 2012; 5:492-497.

70. Margue C, Reinsbach S, Philippidou D, Beaume N, Walters C, Schneider JG, Nashan D, Behrmann I, Kreis S. Comparison of a healthy miRNome with melanoma patient miRNomes: are microRNAs suitable serum biomarkers for cancer? Oncotarget. 2015; 6:12110-12127. doi: 10.18632/oncotarget.3661.

71. Edge SB, Compton CC. The American Joint Committee on Cancer: the 7th edition of the AJCC cancer staging manual and the future of TNM. Ann Surg Oncol. 2010; 17:1471-1474. 
72. Barry SE, Chan B, Ellis M, Yang Y, Plit ML, Guan G, Wang X, Britton WJ, Saunders BM. Identification of miR-93 as a suitable miR for normalizing miRNA in plasma of tuberculosis patients. J Cell Mol Med. 2015; 19:1606-1613.
73. Hanley JA, McNeil BJ. A method of comparing the areas under receiver operating characteristic curves derived from the same cases. Radiology. 1983; 148:839-843.

74. Kaplan EL, Meier P. Nonparametric estimation from in complete observations. J Amer Stat Assoc. 1958; 53:457-481. 